\title{
RASSF Signalling and DNA Damage: Monitoring the Integrity of the Genome?
}

\author{
Simon F. Scrace and Eric O'Neill \\ Department of Oncology, The Gray Institute, University of Oxford, Roosevelt Drive, Oxford OX3 7DQ, UK \\ Correspondence should be addressed to Eric O’Neill, eric.oneill@oncology.ox.ac.uk
}

Received 3 November 2011; Accepted 27 January 2012

Academic Editor: Geoffrey J. Clark

Copyright ( $) 2012$ S. F. Scrace and E. O'Neill. This is an open access article distributed under the Creative Commons Attribution License, which permits unrestricted use, distribution, and reproduction in any medium, provided the original work is properly cited.

\begin{abstract}
The RASSF family of proteins has been extensively studied in terms of their genetics, structure and function. One of the functions that has been increasingly studied is the role of the RASSF proteins in the DNA damage response. Surprisingly, this research, which encompasses both the classical and N-terminal RASSF proteins, has revealed an involvement of the RASSFs in oncogenic pathways as well as the more familiar tumour suppressor pathways usually associated with the RASSF family members. The most studied protein with respect to DNA damage is RASSF1A, which has been shown, not only to be activated by ATM, a major regulator of the DNA damage response, but also to bind to and activate a number of different pathways which all lead to and feedback from the guardian of the genome, p53. In this review we discuss the latest research linking the RASSF proteins to DNA damage signalling and maintenance of genomic integrity and look at how this knowledge is being utilised in the clinic to enhance the effectiveness of traditional cancer therapies such as radiotherapy.
\end{abstract}

\section{Introduction}

RASSF proteins were originally designated on the basis of sequence homology to domains that associate with Ras-like small GTP-binding proteins. These domains are known as Ras association (RA) domains [RalGDS (Ral guanine nucleotide dissociation stimulator)/AF6 (ALL-1 fusion partner from chromosome 6)] and are distinct from Ras-binding domains (RBD) which bind an alternative set of Ras effectors $[1,2]$. Ras belongs to a family of small G-proteins that are ubiquitously expressed and oscillate between an inactive, GDP-bound state, and an active, GTP-bound state, in response to diverse cellular signals. Various GTP-bound Raslike proteins bind effector proteins to mediate distinct biological responses. There are 150 Ras-like proteins encoded in the human genome which can be grouped by homology or functionality, as being similar to Ras, Rho, Rab, Arf (ADPribosylation factor), or Ran. While originally suggested to associate with Ras [3], the RASSF family has a differential affinity for Ras-like GTPases, with NORE1 (RAPL/RASSF5) displaying a much greater affinity for the closely related Ras homolog, Rap1B, than H-Ras itself [4]. The RA domain of RASSF1 associates with K-Ras, rather than $\mathrm{H}$-Ras or N-Ras and is also described to associate with Ran $[5,6]$. There are now 10 members in the RASSF family (RASSF1-10) subdivided into two distinct subgroups, the classical RASSF proteins (RASSF1-6) and the N-terminal RASSF proteins (RASSF7-10) based on the location of the RA domain [7]. Little is known about the GTP-binding proteins that may interact with the majority of the RASSF family or how they function but the potential exists for a greater number of signalling connections. In addition to an RA domain, the classical RASSF proteins also have a protein-protein interaction motif known as the SARAH domain that is responsible for scaffolding and regulatory interactions [8]. This domain is a short coiled-coil region and so named due to its location in the extreme C-terminus of genetically linked Drosophila proteins; Salvador (hSav1/WW45), dRASSF and Hippo (hMST1/2) (SARAH: SAlvador, RAssf, Hippo) which can form both homo- and heterodimers [9]. The N-terminal RASSFs lack an identifiable SARAH domain, although the SMART database predicts that RASSF7, 8 and 10 contain extensive coiled-coil regions, which can dimerise [10].

RASSF1A and RASSF5A [also known as NORE1A (Novel Ras Effector 1 isoform A)] also contain an N-terminal atypical diacylglycerol/phorbol ester-binding (DAG) domain also 
known as the protein kinase $\mathrm{C}$ conserved region (C1) domain that contains a central zinc finger (Zinc-binding domain) [11]. The Zinc finger in the RASSF family members is denoted "atypical" because it lacks critical residues required for binding of phorbol esters or DNA and therefore probably mediates protein-protein interactions. Indeed, structural analysis indicates that the $\mathrm{C} 1$ domain of NORE1A associates with the RA domain to occlude RAS association [12]. As none of the family members have any known enzymatic activity they are thought to be scaffold/adaptor proteins using these binding domains to bring target proteins together to impart their functions.

There are a number of reviews that introduce the RASSF family and the pathways within which they function; however, this paper will focus on the emerging roles of the RASSF family and their effectors in the response to DNA damage. The best described protein in this family with respect to DNA damage is RASSF1 thus the review will concentrate on this protein with particular reference to a recently elucidated signalling network from RASSF1A and the potential clinical significance of targeting this pathway [13].

\section{RASSF1}

It had long been suspected that the 3 p21.3 region of the human genome harboured one or more important tumour suppressors because loss of heterozygosity ( $\mathrm{LOH}$ ) was found at this locus in lung, breast, and kidney tumours and genetic instability in this region is the earliest most frequently detected deficiency in lung cancers [14-20]. This $120 \mathrm{~kb}$ region contains 8 genes namely CACNA2D2, PL6, 101F6, NPRL2/ G21, ZMYND10/BLU, RASSF1/123F2, FUS1, and MYAL2. However, none of these candidate genes are frequently mutated in cancers $[16,21]$. At the same time as these $\mathrm{LOH}$ studies, Dammann et al. identified RASSF1 as an interacting partner of the DNA damage repair protein xeroderma pigmentosum complementation group A (XPA) [22]. While the role of RASSF1 in nucleotide excision repair could not be verified, it may yet prove to be significant given the emerging role of RASSF1 in the DNA damage response. The RASSF1 gene consists of 8 exons spanning a region of about $11 \mathrm{~kb}$. The C-terminal showed high-sequence homology with NORE1, containing an RA domain and thus the gene was named RASSF1 for Ras association domain family member 1 [22]. Alternative splicing generates 8 isoforms $\mathrm{A}-\mathrm{H}$ from promoters held within $2 \mathrm{CpG}$ islands. The first $\mathrm{CpG}$ island encompasses the promoter regions for RASSF1A, D, E, F, and G. Epigenetic inactivation by DNA methylation at this CpG island is one of the most common events in human cancers (reviewed in [23-25]). This methylation has recently been attributed to HOXB3 driven overexpression of the DNA methyltransferase, DNMT3B [26]. RASSF1B, C, and H are generated from a promoter located within the larger $3^{\prime} \mathrm{CpG}$ island [27]. This commonly remains unmethylated in cancers and consequently cells retain expression of these isoforms [23]. RASSF1A and RASSF1C are the major transcripts of the RASSF1 gene and are expressed ubiquitously in normal tissues [28].

\section{RASSF1A}

Exogenous expression of RASSF1A reduces colony formation in soft agar and reduced tumourigenicity in nude mice [22, 29-31]. Similarly, reexpression of RASSF1A using demethyltransferase inhibitors such as zebularine and 5-aza-2' -deoxycytidine caused significant growth arrest in ovarian cancer cell lines [32]. Reciprocally, RASSF1A knockout mice develop spontaneous tumours, particularly when combined with a knockout of p53, highlighting the significance of RASSF1A in tumour development [33-35]. In addition these $\mathrm{RASSF} \mathrm{A}^{-/-}, \mathrm{p} 53^{-/-}$mice showed high levels of aneuploidy/ tetraploidy suggesting an important role for RASSF1A in maintaining genomic integrity. RASSF1A has been shown to have many roles in cell cycle control and microtubule organisation $[23,27]$, the response to DNA damage is, however, only beginning to be elucidated. It is therefore timely to present these pathways and highlight their importance to the DNA damage response, genomic integrity, and cell survival during cancer development.

\section{RASSF1 Phosphorylation}

The majority of the phosphorylation of RASSF1A has being attributed to the phosphorylation of Serine 202/203. These sites have been demonstrated to be targeted by a number of kinases including, both CDK (Cyclin-Dependent Kinase) and Aurora kinases [36-40]. These phosphorylation events prevent the association of RASSF1A with microtubules during prometaphase. The phosphorylation of RASSF1A on these sites also coordinates the regulation of mitosis by controlling activation of the anaphase-promoting complex/ cyclosome (APC/C), and regulation of syntaxin16 to promote cytokinesis $[39,40]$. Loss of phosphorylation at these sites leads to defects in mitosis resulting in aneuploidy and genomic instability.

In the DNA damage response phosphorylation of RASSF1A serine 131 (S131) is emerging as an important phosphorylation site. The initial kinases that respond to breaks in DNA are the phosphatidyl-inositol 3-kinase like kinases ATM (Ataxia Telangiectasia Mutated), ATR (ATMand Rad3-Related), and DNA-PK cs $_{\text {(DNA-dependent protein }}$ kinase catalytic subunit) [41]. RASSF1A has a consensus site for ATM phosphorylation on serine 131 that is conserved in vertebrates and unique amongst family members and has recently been confirmed as a bone-fide target for ATM $[13,42]$. Serine 131 phosphorylation appears important for RASSF1A activation and inactivating mutations of this site have been identified in human cancers [43]. Indeed Shivakumar et al., showed that mutation of the predicted phosphorylation site, S131F, removed the ability to induce cell cycle arrest and block cell proliferation [43]. ATM-dependent phosphorylation at the 131 site is also restricted by S131F and disables the ability of RASSF1A to respond to various DNA-damaging agents [13]. Mutations near the ATM site are hypothesised to function by inactivating ATM phosphorylation. One of these is a nonsynonymous single nucleotide polymorphism (SNP) at p.RASSF1A-A133S (rs2073498), 


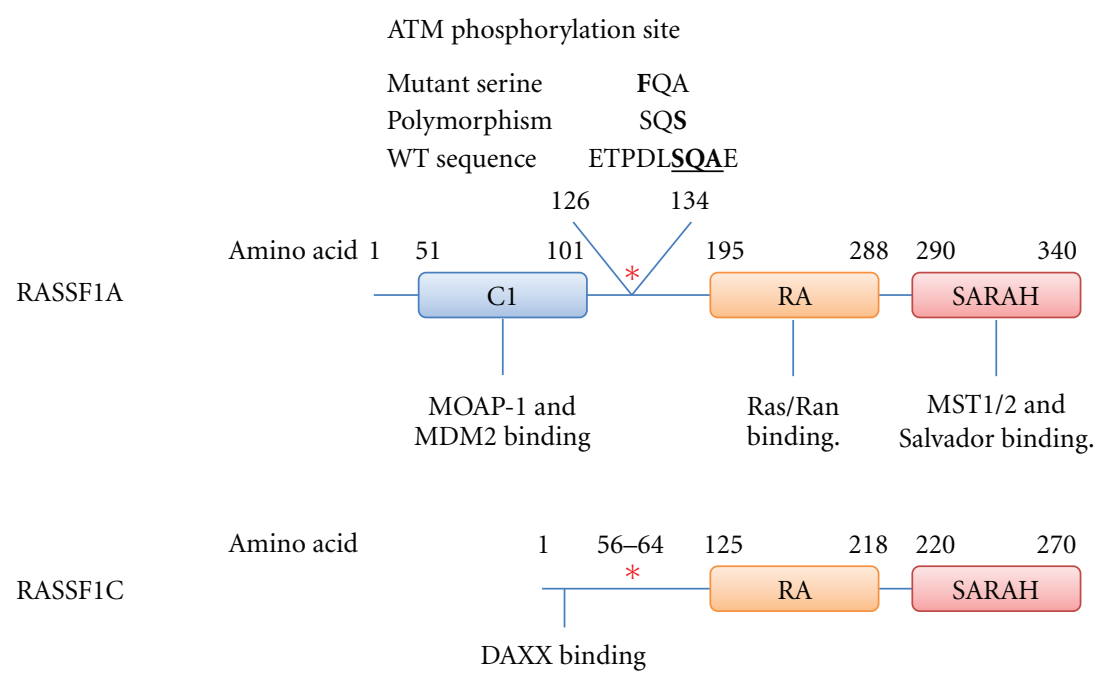

FIgURE 1: Cartoon depicting the interactions of RASSF1A and RASSF1C. RASSF1A and RASSF1C share a common C-terminal aminoacid sequence, which includes the ATM phosphorylation site (red asterisk), the RA domain, and the SARAH domain but differs at the Nterminal. RASSF1A has a C1 domain which interacts with MOAP-1 and MDM2. RASSF1C lacks the C1 domain but has an alternative DAXX interaction domain. Serine 131 of RASSF1A has been shown to be mutated from serine (S) to phenylalanine (F). An alanine (A) to Serine (S) polymorphism also exists at the 133 site.

which has significant allele frequencies in human populations (http://hapmap.ncbi.nlm.nih.gov/). The minor allele of the SNP encodes a serine (A133S) and decreases the ability of RASSF1A to become phosphorylated which, like S131F, results in a defective G1 arrest [43]. This suggests that sequence changes to the ATM consensus sequence (aminoacids 125-138) may severely inhibit the function of RASSF1A by disrupting the phosphorylation of S131 and preventing the activation of RASSF1A (Figure 1).

RASSF1A association with Ran directs the formation of a Ran-GTP gradient between the spindle poles and the metaphase plate which is important for the formation of mitotic spindle and for successful completion of mitosis. RASSF1A targets MST1/2 kinase activity towards the RanGEF (GTP exchange factor) RCC1, which inhibits its function and results in elevated Ran-GTP near the metaphase plate. Taken together, these studies indicate that RASSF1A is important for the maintenance of genomic stability by acting as an integrity checkpoint factor. Loss of RASSF1A is likely to weaken the prometaphase checkpoint and increase the potential to create genomic instability and DNA damage leading to cancer development. Indeed, the restriction of RASSF1A activity by modulation of the ATM site may be linked to numerous observations regarding the early onset of tumours in individuals carrying one minor allele of the p.RASSF1A-A133S polymorphism $[44,45]$. This has been controversially linked to the exacerbation of a BRCA1/2 genomic instability phenotype; however, the inconsistency may be due to confounding factors other than BRCA2 and may be due to genomic instability via defects in RASSF1A itself [46]. All this may indicate that DNA damage activation of RASSF1A may provide an extra level of regulatory response, whereby the prometaphase checkpoint senses cells entering into mitosis with DNA damage.

\section{Regulation by Domain Interaction}

As a scaffold, RASSF1A must exert its tumour suppressor function through its interaction domains. The two most important domains in the context of DNA damage are the C1 domain and the SARAH domain. The most significant binding partners identified to interact with the $\mathrm{C} 1$ domain are the TNF-R1/TRAIL-R1-Modulator of Apoptosis-1 (MOAP-1) complexes and the MDM2/DAXX/HAUSP/p53 complex [47, 48] (Figure 1). MOAP-1 and RASSF1A are recruited to either TNF-R1 or TRAIL-R1 in response to TNF $\alpha$ stimulation. RASSF1A binds MOAP-1 causing an activating conformational change to the structure of MOAP-1. The active structure can bind to the proapoptotic Bcl-2 family member BAX which creates a pore in the outer mitochondrial membrane leading to the release of cytochrome $\mathrm{C}$ and induction of caspase-dependent apoptotic signalling pathways $[47,49]$. BAX and the associated negative regulator BAK tightly regulate the cell's response to apoptotic signals and are often coordinated with other apoptotic signals such as DNA damage. It is reasonable to assume that RASSF1A-MOAP-1 may be affected by DNA damage but whether this contributes to the regulation of BAX/BAK at the mitochondria remains uncertain.

The response of tumour suppressor p53 to DNA damage results in a variety of outcomes including cell cycle arrest, apoptosis, and senescence, combining to protect the integrity of the genome [50,51]. In unstressed cells p53 levels are low, being controlled by the RING domain-containing E3 ubiquitin ligase MDM2 (Mouse Double Minute 2) [52, 53]. Induction of DNA damage results in phosphorylation of $\mathrm{p} 53$ by the DNA damage checkpoint proteins ATM (on serine 15) and CHK2 (Checkpoint Kinase 2) (on serine 20) $[54,55]$. These phosphorylation events combine with an ATM-mediated restriction of MDM2 activity to stabilize p53. Song et al. have 
recently shown that the $\mathrm{C} 1$ domain of RASSF1A can bind and sequester MDM2 in an ATM-dependent manner [48]. They describe a complex consisting of MDM2, DAXX (death-domain-associated protein), and HAUSP1 (a deubiquitinating enzyme). HAUSP1 removes ubiquitin molecules from MDM2 and increases its stability. Upon DNA damage, ATM activates RASSF1A driving its association with MDM2, potentially through phosphorylation at S131. RASSF1A disrupts the MDM2-DAXX-HAUSP1 complex, sequestering MDM2 away from p53, and preventing HAUSP1-regulated deubiquitination of MDM2 promoting its degradation. Release of DAXX from the complex is thought to allow DAXX relocation to the plasma membrane where it can bind the death receptor Fas and activate c-Jun $\mathrm{NH}_{2}$-terminal kinase (JNK) [56]. Activated p53 exerts its tumour suppressor function by acting as a transcription factor. It has recently been shown that the RASSF1 promoter is a target for p53 [57]. Interestingly, p53 appears to downregulate the transcription of RASSF1A hinting at a second mechanism through which p53 can negatively regulate itself in addition to the upregulation of MDM2.

RASSF1A makes two significant interactions through its SARAH domain; the first with mammalian sterile 20-like kinases 1 and 2 (MST1/2) and the second to the scaffold protein Salvador (Figure 1). The RASSF1A interaction with MST1/2 leads to an increase in the local concentration of MST molecules allowing them to undergo transphosphorylation and autoactivation [58]. The interaction further stabilises the MST1/2 kinase activity by preventing dephosphorylation of MST1/2 [59]. MST1/2 were initially cloned from lymphoid cDNA library when looking for human relatives of Saccharomyces cerevisiae protein Ste20 and subsequently shown to be activated by a wide variety of cellular stresses [60-63]. Of note is that both Drosophila dMST (Hippo) and MST2 are activated in response to DNA damage. In mammals, DNA damage induction of MST2 requires direct binding of RASSF1A- and ATM-mediated phosphorylation of S131 $[13,64,65]$. Interestingly MST1 was shown to be able to activate p53 in response to cisplatin-induced DNA damage by phosphorylating and inactivating Sirt1, a deacetylase that inactivates p53 [66]. Additional substrates of MST kinases that may prove subject to DNA damage are the histones $\mathrm{H} 2 \mathrm{~B}$ and H2AX, JNK and FOXO transcription factors [67-70]. However, a clear example of signalling through RASSF1AMST after DNA damage is the recruitment and activation of the large tumour suppressor kinases 1 and 2 (LATS1/2) $[71,72]$.

Studies on the Drosophila homolog of MST1/2, Hippo have discovered that the pathway through Warts (LATS1) is responsible for controlling proliferation and apoptosis and is conserved in both vertebrates and invertebrates. Mutations in pathway member's Hippo (MST1/2), Warts (LATS1/2), Salvador (WW45), or Mats (Mob1 as a tumour suppressor) result in vast tissue overgrowth. The pathway generates a signal to inhibit Yorkie (YAP). Yorkie mutants therefore inevitably show a reduced tissue growth phenotype (reviewed in [73]). Yorkie is a non-DNA binding transcriptional coactivator that binds Scalloped (TEAD1-4) leading to the upregulation of proteins such as Cyclin E and Diap-1 to promote cell division and inhibit apoptosis. In this case Warts phosphorylates Yorkie creating a site for 14-3-3 binding. This sequesters Yorkie in the cytoplasm inhibiting its oncogenic activity [74]. In mammals, in the presence of RASSF1A and a DNA damage signal, LATS1 phosphorylation of YAP maintains a pool of YAP in the nucleus which switches binding partner from the antiapoptotic, YAP-TEAD complex to a proapoptotic YAP-p73 complex [75]. The interaction between YAP1 and p73 stabilises p73 by preventing its nuclear export and subsequent degradation [76-78]. YAP1 functions as a coactivator of p73 and this complex upregulates $\mathrm{p} 73$ responsive genes such as the proapoptotic $\mathrm{BH} 3$ only Bcl-2 family member, PUMA $[79,80]$. This idea is in agreement with the finding that both LATS1 and LATS2 mediate apoptosis through p53. In certain cases LATS2-mediated apoptosis is p53 independent, potentially indicating a switch to YAP1 and p73 [13, 81, 82].

LATS2 has been shown to activate p53 both directly, by binding to and inhibiting MDM2 and indirectly by driving the nuclear accumulation of ASPP1 (apoptosis-stimulating protein of p53) [83, 84]. Interestingly, cytoplasmic ASPP1 appears to behave in an opposite manner and inactivates the ability of LATS1 to interact with YAP1 [85]. As RASSF1A activates LATS1/2 in response to DNA damage this could potentially drive ASPP1 activation of p53 and contribute to the overall p53 response. Interestingly the Drosophila ASPP protein (dASPP) has also been shown to interact with dRASSF8 to regulate C-terminal Src kinase (dCsk) and adherens junctions [86], a site key to the regulation of the core hippo pathway [87].

LATS2 has been implicated in the G1 tetraploidy checkpoint, a process that is thought to be driven by LATS2 activation by ATR and leads to direct stabilisation of p53 [83, 88]. Active p53 then creates a positive feedback loop with LATS2 by upregulating its activity further [88]. In response to UV radiation $\mathrm{CHK} 1$ activation by ATR has been shown to activate LATS2 [89].

Although not addressed in a RASSF1A-dependent manner, YAP forms an additional DNA damage promoted complex with the transcription factor early growth response 1 (EGR1) [90]. The interaction promotes enhanced transcriptional activity of EGR1 for the Bcl-2-associated X (BAX) promoter. Thus YAP can act as an oncogene and a tumour suppressor in a RASSF1A-context-dependent manner.

In Drosophila dRASSF and Salvador are known to com pete for MST binding. Here Salvador acts as an adaptor to bring Hippo and Warts together to activate the hippo pathway, which is antagonised by dRASSF [91]. In mammals, however, RASSF1A can bind both MST1/2 and Salvador at the same time using different regions with the SARAH domain. Using an L308P mutant of RASSF1A that cannot bind MST but remains bound to Salvador, Donninger et al. have shown that the RASSF1A Salvador interaction can activate p73 in an MST-independent manner [92].

\section{RASSF1C}

RASSF1C is the second ubiquitously expressed isoform of the RASSF1 gene. Like RASSF1A, RASSF1C contains the ATM 
consensus sequence (Figure 1). This site, at Serine 61, has not yet been confirmed but the sequence is identical between RASSF1A and RASSF1C at this site so it is plausible to suggest that RASSF1C is also phosphorylated and activated by ATM. Indeed, the Serine 61 to phenylalanine (S61F) mutant of RASSF1C was unable to block the genomic destabilising effects of Ras which can be ablated by overexpression of wildtype RASSF1C in the embryonic kidney cell line 293T and human lung tumour cell line NCI-H1299 [93]. This suggests that DNA damage activation of RASSF1C may require phosphorylation of Serine 61 (RASSF1A-131) site. Further to this, RASSF1C has recently been implicated in a DNA damage response pathway involving DAXX (which also binds to RASSF1A) and JNK [94] (Figure 1). In unstressed conditions RASSF1C is shown to be in a complex with DAXX in the nucleus, recently resolved by NMR [95]. Upon ultraviolet radiation or MMS-induced DNA damage this interaction is lost allowing RASSF1C to move to the cytoplasm where it aids the activation of SAPK/JNK signalling [94]. DAXX, however, remains in the nucleus concentrating at PML bodies. The signal that leads to release of RASSF1C from DAXX is unknown; however, it would be interesting to see if the signal relies upon the ATM phosphorylation site. Conversely, another study has identified that RASSF1C, far from being activated by DNA damage, is targeted for degradation under stress conditions. Exposure to UV radiation or treatment of cells with doxorubicin leads to RASSF1C phosphorylation by GSK $3 \beta$ creating a phosphodegron at S19/23 which is bound to by SCF ${ }^{\beta-T r C P}$ targeting RASSF1C for degradation [96]. This GSK3 $\beta$-dependent degradation was shown to be inhibited by the PI3-K/AKT pathway. Since AKT activity can lead to RASSF1C upregulation it suggests that RASSF1C could function as an oncogene. This is in keeping with several recent reports showing that RASSF1C increased cell proliferation in lung cancer cells and migration in breast cancer cell lines $[97,98]$.

\section{Therapeutic Implications of RASSF1A Loss}

One of the most common and widespread events to occur during cancer development is the loss of RASSF1A expression. This loss is due to methylation of the upstream CpG islands in the RASSF1 gene [22, 29]. The frequency of epigenetically driven loss of RASSF1A correlates well with the increasing grade of the tumour. Methylation has been reported in over 37 tumour types (comprehensively reviewed in $[24,99]$ ) and is thought to be an early event in breast and thyroid tumourigenesis, childhood neoplasia, and endometrial carcinogenesis [27].

RASSF1A methylation correlates with a decreased responsiveness to DNA-damaging therapies [100-102]. The DNA methyltransferase (DNMT) inhibitor zebularine has been used to effectively reexpress RASSF1A and show an increase in cancer cell sensitivity to radiation-induced damage in vitro and in vivo [101] as well as to cisplatin [32]. Dote et al. showed that $48 \mathrm{~h}$ treatment with zebularine, which corresponded to the maximum reexpression of RASSF1A increased the radiosensitivity of $\mathrm{PaCa}, \mathrm{DU} 145$, and U251 cancer cell lines by 1.5 times and caused an increased tumour delay in U251 xenograph models in mice [101]. A $48 \mathrm{~h}$ treatment with zebularine also increased cancer cell sensitivity to DNA damage and a 16-fold reduction in $\mathrm{IC}_{50}$ of cisplatin in resistant ovarian cancer cell lines [32]. Sensitivity of testicular germ cell tumours to cisplatin could also be enhanced by another DNMT inhibitor that is in clinical trials, 5-aza-2' deoxycytidine [103]. Interestingly, they noted that effectiveness of the 5-aza-2' -deoxycytidine treatment was dependent on the level of DNMT3B levels. The higher the DNMT3B level the greater the effect. The most significant target gene for DNMT3B was shown to be RASSF1A (as mentioned above) and thus it can be extrapolated that the increase in sensitivity to cisplatin is due to the reexpression of RASSF1A. Reexpression of RASSF1A using 5-aza-2'-deoxycytidine or reintroduction of RASSF1A into the hepatocellular carcinoma cell line, SMMC-7721, was also shown to increase sensitivity to chemotherapeutics such as fluorouracil, mitomycin, and cisplatin [104]. Together these results support a clinically relevant role for RASSF1A in the DNA damage response that is backed up by phase I and II clinical trials in myelodysplasia and leukaemia patients where 5-aza- $2^{\prime}$-deoxycytidine has shown efficacy both alone and in combination with the histone deacetylase (HDAC) inhibitor valproic acid $[105,106]$. Therapeutic failure upon RASSF1A loss can also be counteracted by targeting the downstream DNA damage responsive signalling pathway. Direct activation of BAX via the $\mathrm{BH} 3$ mimetic ABT-737 has recently put forward as a potential treatment for RASSF1A methylated medulloblastoma [107]. The role of RASSF1A in checkpoint activation and maintenance of genomic integrity is highlighted in a study by Zhang et al. which showed a significant increase in DNA damage caused by aflatoxin $B_{1}$ in tumour tissues where RASSF1A has been lost due to DNA methylation [108].

\section{Other RASSFs and DNA Damage}

This paper has concentrated primarily upon the role of RASSF1 in DNA damage; however, it is worth noting that other RASSF proteins have also been linked to DNA damage pathways. The RASSF2 gene resides on chromosome 20. The gene can be spliced into two very similar proteins RASSF2A and RASSF2C both of which contain the RA domain and the SARAH domain. They show 28\% identity to RASSF1A and like RASSF1A, the promoter has been shown to be inactivated by hypermethylation in primary tumours [109-114]. RASSF2 has been reported to be upregulated in lymphocytes from individuals exposed to ionising radiation [115]. RASSF2 has also been shown to associate with, and is phosphorylated by, MST2 leading to stabilisation of MST2 and the generation of proapoptotic signals [116].

The RASSF6 gene is located on chromosome 4. While the expression of RASSF6 is lost in cancer, in silico analysis did not find any CpG islands located near the promoter; therefore, it is assumed that this loss is not due to DNA methylation $[117,118]$. RASSF6 is known to activate apoptosis in both caspase-dependent and -independent mechanisms in response to TNF $\alpha$; however, it is unknown whether it is also 


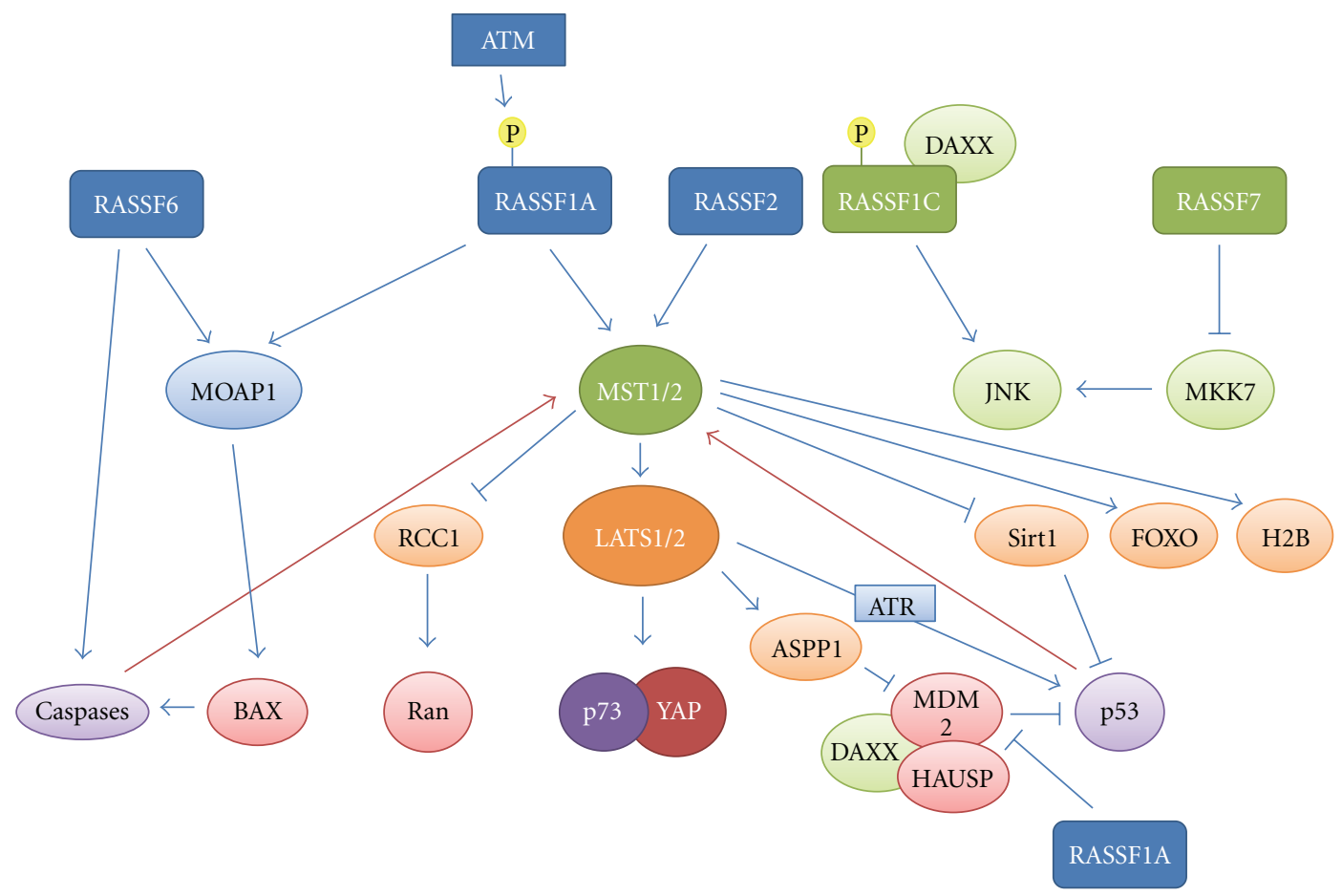

FIGURE 2: Cartoon depicting DNA damage activated pathways downstream of RASSF family members. RASSF family members, activated by DNA damage, signal through various intermediates (primary interaction: light blue and green [involving RASSF1C or RASSF7]; secondary: orange and tertiary: red) to activate p53, p73, and caspases (purple) to control apoptosis, genome stability, and senescence. Feedback loops exist from caspases and p53 that further activate the pathways and amplify the signal. RASSF1A can also directly sequester MDM2 leading to 53 activation. RASSF1C can transfer DNA damage signals from the nucleus to the cytoplasm by activating JNK signalling. RASSF7 acts as an oncogene inhibiting the activation of JNK.

activated by DNA damage signals [118]. RASSF6 contains both the RA domain and SARAH domain and like RASSF1A it has been shown to bind to MOAP-1 [117], which could be responsible for its induction of apoptosis in response to TNF $\alpha$. Unlike other family members, RASSF6 contains a number of ATM consensus sites (SQ/TQ) upstream of the RA domain; however it is not clear if these are functional.

RASSF family members efficiently form heterodimers [119]. This provides a potential mechanism through which additional RASSF proteins could be involved in DNA damage signalling. A heterodimer between RASSF1A and RASSF5A has been suggested to be important for the interaction of RASSF1A with Ras [120]. Given that each of the RASSF proteins above is thought to impart its tumour suppressor function through the MST kinases we could propose that heterodimeric interactions between RASSF family members may be important for their DNA damage-induced apoptotic signalling.

RASSF7 is the best studied N-terminal RASSF protein and the first to be shown to be linked to the DNA damage response. Located on chromosome 11 close to the H-Ras gene (HRAS1), it forms part of a microsatellite that is associated with increased cancer risk [121-123]. Unlike the majority of the RASSF family members that are silenced in cancer, RASSF7 has been shown to be upregulated in a number of cancers including pancreatic, endometrial, and ovarian [124-128]. The upregulation of RASSF7 in cancers suggests an oncogenic function, the mechanism of which has only just started to be explored. RASSF7, in concert with N-Ras, is thought to suppress the activation of JNK in response to low doses of UV radiation by binding and inhibiting MKK7, preventing its interaction with JNK. At higher doses of UV, RASSF7, like RASSF1C, is targeted for degradation through an ubiquitin-dependent mechanism. This frees MKK7 to activate a stress response through JNK [129].

\section{Conclusion}

Ras-association domain containing family members are important tumour suppressors involved in linking cellular stresses to cell cycle arrest and apoptosis (Figure 2). RASSF1A is an adaptor protein with three major interaction domains through which it imparts its functions. Each of these domains is involved in binding different effector proteins in response to DNA damage. The C1 domain binds MDM2 to stabilise p53 and the RA and SARAH domains are required to activate the mammalian Hippo pathway. The mammalian homolog of Hippo, MST1/2, can activate apoptosis in response to cellular stresses either directly, in the case of FOXO1 and histone H2B or via LATS1/2. RASSF2, RASSF5, and RASSF6 which share the RA and SARAH domains with RASSF1A have also been shown to active MST1/2 to induce 
apoptosis as well as being able to induce apoptosis independently of the Hippo pathway. LATS1 and 2 have been implicated in apoptosis by stabilising both p53, either directly through an interaction with MDM2 or indirectly via ASPP1 and stabilising p73 via YAP, in response to DNA damage. RASSF1C has been shown to be released from DAXX and p53 upon DNA damage where it can go and transmit the damage signal from the nucleus to the cytoplasm by activating JNK signalling. Each of these proteins appears to act both upstream and downstream of the "guardian of the genome" p53 to create a network which feeds back upon itself to enhance the DNA damage signaling within the cell. Greater than $50 \%$ of human tumours has either lost or mutated p53. Disruption of these networks will inactivate p53 and may contribute to tumourigenesis in a number of the cases where wild-type p53 is retained. Although not correlated with p53 loss or mutation, RASSF proteins are epigenetically lost in human cancers by DNA methylation. It has been shown that, as with p53, loss of RASSF1 expression is associated with more aggressive tumours and increased resistance to radiation-induced DNA damage and platinum-based drugs. DMNT inhibitors such as zebularine have been shown to reexpress RASSF1A and increase the radiosensitivity of these cancers suggesting that reexpression of RASSF1A and other silenced RASSFs maybe a path through which chemoradioresistant tumours can be combated.

\section{References}

[1] C. P. Ponting and D. R. Benjamin, "A novel family of Rasbinding domains," Trends in Biochemical Sciences, vol. 21, no. 11, pp. 422-425, 1996.

[2] T. Yamamoto, S. Taya, and K. Kaibuchi, "Ras-induced transformation and signaling pathway," Journal of Biochemistry, vol. 126, no. 5, pp. 799-803, 1999.

[3] D. Vavvas, X. Li, J. Avruch, and X. F. Zhang, "Identification of Nore1 as a potential Ras effector," Journal of Biological Chemistry, vol. 273, no. 10, pp. 5439-5442, 1998.

[4] S. Wohlgemuth, C. Kiel, A. Krämer, L. Serrano, F. Wittinghofer, and C. Herrmann, "Recognizing and defining true ras binding domains I: biochemical analysis," Journal of Molecular Biology, vol. 348, no. 3, pp. 741-758, 2005.

[5] A. Dallol, L. B. Hesson, D. Matallanas et al., "RAN GTPase is a RASSF1A effector involved in controlling microtubule organization," Current Biology, vol. 19, no. 14, pp. 1227 $1232,2009$.

[6] D. Matallanas, D. Romano, F. Al-Mulla et al., "Mutant K-Ras activation of the proapoptotic MST2 pathway is antagonized by wild-type K-Ras," Molecular Cell, vol. 44, no. 6, pp. 893906, 2011.

[7] V. Sherwood, R. Manbodh, C. Sheppard, and A. D. Chalmers, "RASSF7 is a member of a new family of RAS association domain-containing proteins and is required for completing mitosis," Molecular Biology of the Cell, vol. 19, no. 4, pp. 17721782, 2008.

[8] E. Hwang, K. S. Ryu, K. Pääkkönen et al., "Structural insight into dimeric interaction of the SARAH domains from Mst1 and RASSF family proteins in the apoptosis pathway," Proceedings of the National Academy of Sciences of the United States of America, vol. 104, no. 22, pp. 9236-9241, 2007.
[9] H. Scheel and K. Hofmann, "A novel interaction motif, SARAH, connects three classes of tumor suppressor," Current Biology, vol. 13, no. 23, pp. R899-R900, 2003.

[10] G. Grigoryan and A. E. Keating, "Structural specificity in coiled-coil interactions," Current Opinion in Structural Biology, vol. 18, no. 4, pp. 477-483, 2008.

[11] A. C. Newton, "Protein kinase C: seeing two domains," Current Biology, vol. 5, no. 9, pp. 973-976, 1995.

[12] E. Harjes, S. Harjes, S. Wohlgemuth et al., "GTP-Ras disrupts the intramolecular complex of C1 and RA domains of nore1," Structure, vol. 14, no. 5, pp. 881-888, 2006.

[13] G. Hamilton, K. S. Yee, S. Scrace, and E. O’Neill, "ATM regulates a RASSF1A-dependent DNA damage response," Current Biology, vol. 19, no. 23, pp. 2020-2025, 2009.

[14] J. Hung, Y. Kishimoto, K. Sugio et al., "Allele-specific chromosome $3 p$ deletions occur at an early stage in the pathogenesis of lung carcinoma," Journal of the American Medical Association, vol. 273, no. 24, p. 1908, 1995.

[15] K. Kok, S. L. Naylor, and C. H. C. M. Buys, "Deletions of the short arm of chromosome 3 in solid tumors and the search for suppressor genes," Advances in Cancer Research, vol. 71, pp. 27-92, 1997.

[16] Y. Sekido, M. Ahmadian, I. I. Wistuba et al., "Cloning of a breast cancer homozygous deletion junction narrows the region of search for a $3 \mathrm{p} 21.3$ tumor suppressor gene," Oncogene, vol. 16, no. 24, pp. 3151-3157, 1998.

[17] V. Sundaresan, S. P. Ganly, P. Hasleton et al., "p53 and chromosome 3 abnormalities, characteristic of malignant lung tumours, are detectable in preinvasive lesions of the bronchus," Oncogene, vol. 7, no. 10, pp. 1989-1997, 1992.

[18] I. I. Wistuba, C. Behrens, S. Milchgrub et al., "Sequential molecular abnormalities are involved in the multistage development of squamous cell lung carcinoma," Oncogene, vol. 18, no. 3, pp. 643-650, 1999.

[19] I. I. Wistuba, C. Behrens, A. K. Virmani et al., "High resolution chromosome $3 p$ allelotyping of human lung cancer and preneoplastic/preinvasive bronchial epithelium reveals multiple, discontinuous sites of $3 p$ allele loss and three regions of frequent breakpoints," Cancer Research, vol. 60, no. 7, pp. 1949-1960, 2000.

[20] Y. Sekido, K. M. Fong, and J. D. Minna, "Progress in understanding the molecular pathogenesis of human lung cancer," Biochimica et Biophysica Acta, vol. 1378, no. 1, pp. F21-F59, 1998.

[21] M. I. Lerman and J. D. Minna, “The 630-kb lung cancer homozygous deletion region on human chromosome 3 p21.3: identification and evaluation of the resident candidate tumor suppressor genes," Cancer Research, vol. 60, no. 21, pp. 61166133, 2000.

[22] R. Dammann, C. Li, J. H. Yoon, P. L. Chin, S. Bates, and G. P. Pfeifer, "Epigenetic inactivation of a RAS association domain family protein from the lung tumour suppressor locus 3p21.3," Nature Genetics, vol. 25, no. 3, pp. 315-319, 2000.

[23] H. Donninger, M. D. Vos, and G. J. Clark, "The RASSF1A tumor suppressor," Journal of Cell Science, vol. 120, no. 18, pp. 3163-3172, 2007.

[24] L. B. Hesson, W. N. Cooper, and F. Latif, "The role of RASSF1A methylation in cancer," Disease Markers, vol. 23, no. 1-2, pp. 73-87, 2007.

[25] P. A. Jones and S. B. Baylin, "The epigenomics of cancer," Cell, vol. 128, no. 4, pp. 683-692, 2007.

[26] R. K. Palakurthy, N. Wajapeyee, M. K. Santra et al., "Epigenetic silencing of the RASSF1A tumor suppressor gene 
through HOXB3-mediated induction of DNMT3B expression," Molecular Cell, vol. 36, no. 2, pp. 219-230, 2009.

[27] L. van der Weyden and D. J. Adams, "The Ras-association domain family (RASSF) members and their role in human tumourigenesis," Biochimica et Biophysica Acta, vol. 1776, no. 1, pp. 58-85, 2007.

[28] A. M. Richter, G. P. Pfeifer, and R. H. Dammann, "The RASSF proteins in cancer; from epigenetic silencing to functional characterization," Biochimica et Biophysica Acta, vol. 1796, no. 2, pp. 114-128, 2009.

[29] D. G. Burbee, E. Forgacs, S. Zöchbauer-Müller et al., "Epigenetic inactivation of RASSF1A in lung and breast cancers and malignant phenotype suppression," Journal of the National Cancer Institute, vol. 93, no. 9, pp. 691-699, 2001.

[30] K. Dreijerink, E. Braga, I. Kuzmin et al., "The candidate tumor suppressor gene, RASSF1A, from human chromosome 3 p21.3 is involved in kidney tumorigenesis," Proceedings of the National Academy of Sciences of the United States of America, vol. 98, no. 13, pp. 7504-7509, 2001.

[31] I. Kuzmin, J. W. Gillespie, A. Protopopov et al., "The RASSF1A tumor suppressor gene is inactivated in prostate tumors and suppresses growth of prostate carcinoma cells," Cancer Research, vol. 62, no. 12, pp. 3498-3502, 2002.

[32] C. Balch, P. Yan, T. Craft et al., "Antimitogenic and chemosensitizing effects of the methylation inhibitor zebularine in ovarian cancer," Molecular Cancer Therapeutics, vol. 4, no. 10, pp. 1505-1514, 2005.

[33] S. Tommasi, R. Dammann, Z. Zhang et al., "Tumor susceptibility of Rassfla knockout mice," Cancer Research, vol. 65, no. 1, pp. 92-98, 2005.

[34] L. Van Der Weyden, K. K. Tachibana, M. A. Gonzalez et al., "The RASSF1A isoform of RASSF1 promotes microtubule stability and suppresses tumorigenesis," Molecular and Cellular Biology, vol. 25, no. 18, pp. 8356-8367, 2005.

[35] S. Tommasi, A. Besaratinia, S. P. Wilczynski, and G. P. Pfeifer, "Loss of Rassfla enhances p53-mediated tumor predisposition and accelerates progression to aneuploidy," Oncogene, vol. 30, no. 6, pp. 690-700, 2011.

[36] L. Liu, C. Guo, R. Dammann, S. Tommasi, and G. P. Pfeifer, "RASSF1A interacts with and activates the mitotic kinase Aurora-A," Oncogene, vol. 27, no. 47, pp. 6175-6186, 2008.

[37] R. Rong, L. Y. Jiang, M. S. Sheikh, and Y. Huang, "Mitotic kinase Aurora-A phosphorylates RASSF1A and modulates RASSF1A-mediated microtubule interaction and M-phase cell cycle regulation," Oncogene, vol. 26, no. 55, pp. 7700 7708, 2007.

[38] M. S. Song, S. J. Song, S. J. Kim, K. Nakayama, K. I. Nakayama, and D. S. Lim, "Skp2 regulates the antiproliferative function of the tumor suppressor RASSF1A via ubiquitin-mediated degradation at the G1-S transition," Oncogene, vol. 27, no. 22, pp. 3176-3185, 2008.

[39] S. J. Song, S. J. Kim, M. S. Song, and D. S. Lim, "Aurora B-mediated phosphorylation of RASSF1A maintains proper cytokinesis by recruiting syntaxin 16 to the midzone and midbody," Cancer Research, vol. 69, no. 22, pp. 8540-8544, 2009.

[40] S. J. Song, M. S. Song, S. J. Kim et al., "Aurora a regulates prometaphase progression by inhibiting the ability of RASSF1A to suppress APC-Cdc20 activity," Cancer Research, vol. 69, no. 6, pp. 2314-2323, 2009.

[41] D. Durocher and S. P. Jackson, "DNA-PK, ATM and ATR as sensors of DNA damage: variations on a theme?" Current Opinion in Cell Biology, vol. 13, no. 2, pp. 225-231, 2001.

[42] S. T. Kim, D. S. Lim, C. E. Canman, and M. B. Kastan, "Substrate specificities and identification of putative substrates of
ATM kinase family members," Journal of Biological Chemistry, vol. 274, no. 53, pp. 37538-37543, 1999.

[43] L. Shivakumar, J. Minna, T. Sakamaki, R. Pestell, and M. A. White, "The RASSF1A tumor suppressor blocks cell cycle progression and inhibits cyclin D1 accumulation," Molecular and Cellular Biology, vol. 22, no. 12, pp. 4309-4318, 2002.

[44] H. Endoh, Y. Yatabe, S. Shimizu et al., "RASSF1A gene inactivation in non-small cell lung cancer and its clinical implication," International Journal of Cancer, vol. 106, no. 1, pp. 45$51,2003$.

[45] B. Gao, X. J. Xie, C. Huang et al., "RASSF1A polymorphism A133S is associated with early onset breast cancer in BRCA1/2 mutation carriers," Cancer Research, vol. 68, no. 1, pp. 22-25, 2008.

[46] J. Bergqvist, A. Latif, S. A. Roberts et al., "RASSF1A polymorphism in familial breast cancer," Familial Cancer, vol. 9, no. 3, pp. 263-265, 2010.

[47] C. J. Foley, H. Freedman, S. L. Choo et al., "Dynamics of RASSF1A/MOAP-1 association with death receptors," Molecular and Cellular Biology, vol. 28, no. 14, pp. 4520-4535, 2008.

[48] M. S. Song, S. J. Song, S. Y. Kim, H. J. Oh, and D. S. Lim, "The tumour suppressor RASSF1A promotes MDM2 selfubiquitination by disrupting the MDM2-DAXX-HAUSP complex," EMBO Journal, vol. 27, no. 13, pp. 1863-1874, 2008.

[49] S. Baksh, S. Tommasi, S. Fenton et al., "The tumor suppressor RASSF1A and MAP-1 link death receptor signaling to bax conformational change and cell death," Molecular Cell, vol. 18, no. 6, pp. 637-650, 2005.

[50] D. Michael and M. Oren, "The p53-Mdm2 module and the ubiquitin system," Seminars in Cancer Biology, vol. 13, no. 1, pp. 49-58, 2003.

[51] B. Vogelstein, D. Lane, and A. J. Levine, "Surfing the p53 network," Nature, vol. 408, no. 6810, pp. 307-310, 2000.

[52] Y. Haupt, R. Maya, A. Kazaz, and M. Oren, "Mdm2 promotes the rapid degradation of p53," Nature, vol. 387, no. 6630, pp. 296-299, 1997.

[53] M. H. G. Kubbutat, S. N. Jones, and K. H. Vousden, "Regulation of p53 stability by Mdm2," Nature, vol. 387, no. 6630, pp. 299-303, 1997.

[54] A. M. Bode and Z. Dong, "Post-translational modification of p53 in tumorigenesis," Nature Reviews Cancer, vol. 4, no. 10, pp. 793-805, 2004.

[55] R. Maya, M. Balass, S. T. Kim et al., "ATM-dependent phosphorylation of Mdm2 on serine 395: role in p53 activation by DNA damage," Genes and Development, vol. 15, no. 9, pp. 1067-1077, 2001.

[56] X. Yang, R. Khosravi-Far, H. Y. Chang, and D. Baltimore, "Daxx, a novel fas-binding protein that activates JNK and apoptosis," Cell, vol. 89, no. 7, pp. 1067-1076, 1997.

[57] Y. Tian et al., "Tumor suppressor RASSF1A promoter: p53 binding and methylation," PLoS One, vol. 6, no. 3, article e17017, 2011.

[58] H. Glantschnig, G. A. Rodan, and A. A. Reszka, "Mapping of MST1 kinase sites of phosphorylation: activation and autophosphorylation," Journal of Biological Chemistry, vol. 277, no. 45, pp. 42987-42996, 2002.

[59] C. Guo, X. Zhang, and G. P. Pfeifer, "The tumor suppressor RASSF1A prevents dephosphorylation of the mammalian STE20-like kinases MST1 and MST2," Journal of Biological Chemistry, vol. 286, no. 8, pp. 6253-6261, 2011.

[60] C. L. Creasy, D. M. Ambrose, and J. Chernoff, "The Ste20-like protein kinase, Mst1, dimerizes and contains an inhibitory 
domain," Journal of Biological Chemistry, vol. 271, no. 35, pp. 21049-21053, 1996.

[61] J. Chernoff, "Cloning and characterization of a member of the MST subfamily of Ste20-like kinases," Gene, vol. 167, no. 1-2, pp. 303-306, 1995.

[62] C. L. Creasy and J. Chernoff, "Cloning and characterization of a human protein kinase with homology to Ste20," Journal of Biological Chemistry, vol. 270, no. 37, pp. 21695-21700, 1995.

[63] L. K. Taylor, H. C. R. Wang, and R. L. Erikson, "Newly identified stress-responsive protein kinases, Krs-1 and Krs-2," Proceedings of the National Academy of Sciences of the United States of America, vol. 93, no. 19, pp. 10099-10104, 1996.

[64] J. Colombani, C. Polesello, F. Josué, and N. Tapon, "Dmp53 activates the hippo pathway to promote cell death in response to DNA damage," Current Biology, vol. 16, no. 14, pp. 1453 1458, 2006.

[65] W. Wen, F. Zhu, J. Zhang et al., "MST1 promotes apoptosis through phosphorylation of histone H2AX," Journal of Biological Chemistry, vol. 285, no. 50, pp. 39108-39116, 2010.

[66] F. Yuan, Q. Xie, J. Wu et al., "MST1 promotes apoptosis through regulating Sirt1-dependent p53 deacetylation," Journal of Biological Chemistry, vol. 286, no. 9, pp. 6940-6945, 2011.

[67] W. L. Cheung, K. Ajiro, K. Samejima et al., "Apoptotic phosphorylation of histone $\mathrm{H} 2 \mathrm{~B}$ is mediated by mammalian sterile twenty kinase," Cell, vol. 113, no. 4, pp. 507-517, 2003.

[68] M. K. Lehtinen, Z. Yuan, P. R. Boag et al., "A conserved MSTFOXO signaling pathway mediates oxidative-stress responses and extends life span," Cell, vol. 125, no. 5, pp. 987-1001, 2006.

[69] S. Ura, H. Nishina, Y. Gotoh, and T. Katada, "Activation of the c-Jun N-terminal kinase pathway by MST1 is essential and sufficient for the induction of chromatin condensation during apoptosis," Molecular and Cellular Biology, vol. 27, no. 15, pp. 5514-5522, 2007.

[70] M. Praskova, F. Xia, and J. Avruch, "MOBKL1A/MOBKL1B phosphorylation by MST1 and MST2 inhibits cell proliferation," Current Biology, vol. 18, no. 5, pp. 311-321, 2008.

[71] C. Guo, S. Tommasi, L. Liu, J. K. Yee, R. Dammann, and G. Pfeifer, "RASSF1A is part of a complex similar to the drosophila hippo/salvador/lats tumor-suppressor network," Current Biology, vol. 17, no. 8, pp. 700-705, 2007.

[72] E. E. O’Neill, D. Matallanas, and W. Kolch, "Mammalian sterile 20-like kinases in tumor suppression: an emerging pathway," Cancer Research, vol. 65, no. 13, pp. 5485-5487, 2005.

[73] B. V. V. G. Reddy and K. D. Irvine, "The fat and warts signaling pathways: new insights into their regulation, mechanism and conservation," Development, vol. 135, no. 17, pp. 28272838, 2008.

[74] B. Zhao, X. Wei, W. Li et al., "Inactivation of YAP oncoprotein by the Hippo pathway is involved in cell contact inhibition and tissue growth control," Genes and Development, vol. 21, no. 21, pp. 2747-2761, 2007.

[75] K. S. Yee and E. O’Neill, "YAP1: friend and foe," Cell Cycle, vol. 9, no. 8, pp. 1447-1448, 2010.

[76] S. Basu, N. F. Totty, M. S. Irwin, M. Sudol, and J. Downward, "Akt phosphorylates the Yes-associated protein, YAP, to induce interaction with 14-3-3 and attenuation of p73-mediated apoptosis," Molecular Cell, vol. 11, no. 1, pp. 11-23, 2003.

[77] M. Dobbelstein, S. Strano, J. Roth, and G. Blandino, "p73induced apoptosis: a question of compartments and cooperation," Biochemical and Biophysical Research Communications, vol. 331, no. 3, pp. 688-693, 2005.
[78] G. Melino, F. Bernassola, M. Ranalli et al., "p75 induces apoptosis via PUMA transactivation and bax mitochondrial translocation," Journal of Biological Chemistry, vol. 279, no. 9, pp. 8076-8083, 2004.

[79] D. Matallanas, D. Romano, K. Yee et al., "RASSF1A elicits apoptosis through an MST2 pathway directing proapoptotic transcription by the $\mathrm{p} 73$ tumor suppressor protein," Molecular Cell, vol. 27, no. 6, pp. 962-975, 2007.

[80] S. Strano, O. Monti, N. Pediconi et al., "The transcriptional coactivator yes-associated protein drives p73 gene-target specificity in response to DNA damage," Molecular Cell, vol. 18, no. 4, pp. 447-459, 2005.

[81] H. Ke, J. Pei, Z. Ni et al., "Putative tumor suppressor Lats2 induces apoptosis through downregulation of Bcl-2 and BclxL," Experimental Cell Research, vol. 298, no. 2, pp. 329-338, 2004.

[82] M. Kawahara, T. Hori, K. Chonabayashi, T. Oka, M. Sudol, and T. Uchiyama, "Kpm/Lats2 is linked to chemosensitivity of leukemic cells through the stabilization of p73," Blood, vol. 112, no. 9, pp. 3856-3866, 2008.

[83] Y. Aylon, N. Yabuta, H. Besserglick et al., "Silencing of the lats2 tumor suppressor overrides a p53-dependent oncogenic stress checkpoint and enables mutant H-Ras-driven cell transformation," Oncogene, vol. 28, no. 50, pp. 4469-4479, 2009.

[84] Y. Aylon, Y. Ofir-Rosenfeld, N. Yabuta et al., "The Lats2 tumor suppressor augments p53-mediated apoptosis by promoting the nuclear proapoptotic function of ASPP1," Genes and Development, vol. 24, no. 21, pp. 2420-2429, 2010.

[85] A. M. Vigneron, R. L. Ludwig, and K. H. Vousden, "Cytoplasmic ASPP1 inhibits apoptosis through the control of YAP," Genes and Development, vol. 24, no. 21, pp. 2430-2439, 2010.

[86] P. F. Langton, J. Colombani, E. H. Y. Chan, A. Wepf, M. Gstaiger, and N. Tapon, "The dASPP-dRASSF8 complex regulates cell-cell adhesion during drosophila retinal morphogenesis," Current Biology, vol. 19, no. 23, pp. 1969-1978, 2009.

[87] D. Pan, "The hippo signaling pathway in development and cancer," Developmental Cell, vol. 19, no. 4, pp. 491-505, 2010.

[88] Y. Aylon, D. Michael, A. Shmueli, N. Yabuta, H. Nojima, and M. Oren, "A positive feedback loop between the p53 and Lats2 tumor suppressors prevents tetraploidization," Genes and Development, vol. 20, no. 19, pp. 2687-2700, 2006.

[89] N. Okada, N. Yabuta, H. Suzuki, Y. Aylon, M. Oren, and H. Nojima, "A novel Chk1/2-Lats2-14-3-3 signaling pathway regulates P-body formation in response to UV damage," Journal of Cell Science, vol. 124, no. 1, pp. 57-67, 2011.

[90] M. Zagurovskaya, M. M. Shareef, A. Das et al., "EGR-1 forms a complex with YAP-1 and upregulates Bax expression in irradiated prostate carcinoma cells," Oncogene, vol. 28, no. 8, pp. 1121-1131, 2009.

[91] C. Polesello, S. Huelsmann, N. Brown, and N. Tapon, "The drosophila RASSF homolog antagonizes the hippo pathway," Current Biology, vol. 16, no. 24, pp. 2459-2465, 2006.

[92] H. Donninger, N. Allen, A. Henson et al., "Salvador protein is a tumor suppressor effector of RASSF1A with hippo pathway-independent functions," Journal of Biological Chemistry, vol. 286, no. 21, pp. 18483-18491, 2011.

[93] M. D. Vos, A. Martinez, C. Elam et al., "A role for the RASSF1A tumor suppressor in the regulation of tubulin polymerization and genomic stability," Cancer Research, vol. 64, no. 12, pp. 4244-4250, 2004.

[94] D. Kitagawa, H. Kajiho, T. Negishi et al., "Release of RASSF1C from the nucleus by Daxx degradation links DNA damage 
and SAPK/JNK activation," EMBO Journal, vol. 25, no. 14, pp. 3286-3297, 2006.

[95] E. Escobar-Cabrera, D. K. W. Lau, S. Giovinazzi, A. M. Ishov, and L. P. McIntosh, "Structural characterization of the DAXX $\mathrm{N}$-terminal helical bundle domain and its complex with Rassf1C," Structure, vol. 18, no. 12, pp. 1642-1653, 2010.

[96] X. Zhou, T.-T. Li, X. Feng et al., "Targeted polyubiquitylation of RASSF1C by the Mule and SCF $\beta$-TrCP ligases in response to DNA damage," Biochemical Journal, vol. 441, no. 1, pp. 227-236, 2012.

[97] Y. G. Amaar, M. G. Minera, L. K. Hatran, D. D. Strong, S. Mohan, and M. E. Reeves, "Ras association domain family $1 \mathrm{C}$ protein stimulates human lung cancer cell proliferation," American Journal of Physiology, vol. 291, no. 6, pp. L1185L1190, 2006.

[98] M. E. Reeves, S. W. Baldwin, M. L. Baldwin et al., "Rasassociation domain family $1 \mathrm{C}$ protein promotes breast cancer cell migration and attenuates apoptosis," BMC Cancer, vol. 10, article 562, 2010.

[99] R. Dammann, U. Schagdarsurengin, C. Seidel et al., "The tumor suppressor RASSF1A in human carcinogenesis: an update," Histology and Histopathology, vol. 20, no. 2, pp. 645663, 2005.

[100] J. W. F. Catto, A. R. Azzouzi, I. Rehman et al., "Promoter hypermethylation is associated with tumor location, stage, and subsequent progression in transitional cell carcinoma," Journal of Clinical Oncology, vol. 23, no. 13, pp. 2903-2910, 2005.

[101] H. Dote, D. Cerna, W. E. Burgan et al., "Enhancement of in vitro and in vivo tumor cell radiosensitivity by the DNA methylation inhibitor zebularine," Clinical Cancer Research, vol. 11, no. 12, pp. 4571-4579, 2005.

[102] S. Honda, M. Haruta, W. Sugawara et al., "The methylation status of RASSF1A promoter predicts responsiveness to chemotherapy and eventual cure in hepatoblastoma patients," International Journal of Cancer, vol. 123, no. 5, pp. 1117-1125, 2008.

[103] M. J. Beyrouthy, K. M. Garner, M. P. Hever et al., "High DNA methyltransferase 3B expression mediates 5-aza-deoxycytidine hypersensitivity in testicular germ cell tumors," Cancer Research, vol. 69, no. 24, pp. 9360-9366, 2009.

[104] Q.-Z. Zhao and K.-F. Dou, "Methylation of Ras association domain family protein 1 , isoform a correlated with proliferation and drug resistance in hepatocellular carcinoma cell line SMMC-7721," Journal of Gastroenterology and Hepatology, vol. 22, no. 5, pp. 683-689, 2007.

[105] G. Garcia-Manero, H. M. Kantarjian, B. Sanchez-Gonzalez et al., "Phase $1 / 2$ study of the combination of 5-aza-2'-deoxycytidine with valproic acid in patients with leukemia," Blood, vol. 108, no. 10, pp. 3271-3279, 2006.

[106] B. Rüter, P. W. Wijermans, and M. Lübbert, "Superiority of prolonged low-dose azanucleoside administration? Results of 5-aza-2'-deoxycytidine retreatment in high-risk myelodysplasia patients," Cancer, vol. 106, no. 8, pp. 1744-1750, 2006.

[107] J. Levesley et al., "RASSF1A and the BH3-only mimetic ABT737 promote apoptosis in pediatric medulloblastoma cell lines," Neuro-Oncology, vol. 13, no. 12, pp. 1265-1276, 2011.

[108] Y. J. Zhang, H. Ahsan, Y. Chen et al., "High frequency of promoter hypermethylation of RASSF1A and p16 and its relationship to aflatoxin B1-DNA adduct levels in human hepatocellular carcinoma," Molecular Carcinogenesis, vol. 35, no. 2, pp. 85-92, 2002.
[109] K. Akino, M. Toyota, H. Suzuki et al., "The Ras effector RASSF2 is a novel tumor-suppressor gene in human colorectal cancer," Gastroenterology, vol. 129, no. 1, pp. 156-169, 2005.

[110] M. Endoh, G. Tamura, T. Honda et al., "RASSF2, a potential tumour suppressor, is silenced by CpG island hypermethylation in gastric cancer," British Journal of Cancer, vol. 93, no. 12, pp. 1395-1399, 2005.

[111] L. B. Hesson, R. Wilson, D. Morton et al., "CpG island promoter hypermethylation of a novel Ras-effector gene RASSF2A is an early event in colon carcinogenesis and correlates inversely with K-ras mutations," Oncogene, vol. 24, no. 24, pp. 3987-3994, 2005.

[112] H. W. Park, C. K. Hio, I. J. Kim et al., "Correlation between hypermethylation of the RASSF2A promoter and Kras/BRAF mutations in microsatellite-stable colorectal cancers," International Journal of Cancer, vol. 120, no. 1, pp. 7$12,2007$.

[113] M. D. Vos, C. A. Ellis, C. Elam, A. S. Ülkü, B. J. Taylor, and G. J. Clark, "RASSF2 is a novel K-Ras-specific effector and potential tumor suppressor," Journal of Biological Chemistry, vol. 278, no. 30, pp. 28045-28051, 2003.

[114] Z. Zhang, D. Sun, N. V. Do, A. Tang, L. Hu, and G. Huang, "Inactivation of RASSF2A by promoter methylation correlates with lymph node metastasis in nasopharyngeal carcinoma," International Journal of Cancer, vol. 120, no. 1, pp. 32-38, 2007.

[115] E. T. Sakamoto-Hojo, S. S. Mello, E. Pereira et al., "Gene expression profiles in human cells submitted to genotoxic stress," Mutation Research - Reviews in Mutation Research, vol. 544, no. 2-3, pp. 403-413, 2003.

[116] W. N. Cooper, L. B. Hesson, D. Matallanas et al., "RASSF2 associates with and stabilizes the proapoptotic kinase MST2," Oncogene, vol. 28, no. 33, pp. 2988-2998, 2009.

[117] N. P. C. Allen, H. Donninger, M. D. Vos et al., "RASSF6 is a novel member of the RASSF family of tumor suppressors," Oncogene, vol. 26, no. 42, pp. 6203-6211, 2007.

[118] M. Ikeda, S. Hirabayashi, N. Fujiwara et al., "Ras-association domain family protein 6 induces apoptosis via both caspasedependent and caspase-independent pathways," Experimental Cell Research, vol. 313, no. 7, pp. 1484-1495, 2007.

[119] M. Ikeda, A. Kawata, M. Nishikawa et al., "Hippo pathwaydependent and-independent roles of RASSF6," Science Signaling, vol. 2, no. 90, article ra59, 2009.

[120] S. Ortiz-Vega, A. Khokhlatchev, M. Nedwidek et al., "The putative tumor suppressor RASSF1A homodimerizes and heterodimerizes with the Ras-GTP binding protein Nore1," Oncogene, vol. 21, no. 9, pp. 1381-1390, 2002.

[121] J. N. Weitzel, A. Kasperczyk, C. Mohan, and T. G. Krontiris, "The HRAS1 gene cluster: two upstream regions recognizing transcripts and a third encoding a gene with a leucine zipper domain," Genomics, vol. 14, no. 2, pp. 309-319, 1992.

[122] T. G. Krontiris, B. Devlin, D. D. Karp, N. J. Robert, and N. Risch, "An association between the risk of cancer and mutations in the HRAS1 minisatellite locus," New England Journal of Medicine, vol. 329, no. 8, pp. 517-523, 1993.

[123] T. G. Krontiris, N. A. DiMartino, M. Colb, and D. R. Parkinson, "Unique allelic restriction fragments of the human Haras locus in leukocyte and tumour DNAs of cancer patients," Nature, vol. 313, no. 6001, pp. 369-374, 1985.

[124] H. Friess, J. Ding, J. Kleeff et al., "Microarray-based identification of differentially expressed growth- and metastasisassociated genes in pancreatic cancer," Cellular and Molecular Life Sciences, vol. 60, no. 6, pp. 1180-1199, 2003. 
[125] C. D. Logsdon, D. M. Simeone, C. Binkley et al., "Molecular profiling of pancreatic adenocarcinoma and chronic pancreatitis identifies multiple genes differentially regulated in pancreatic cancer," Cancer Research, vol. 63, no. 10, pp. 26492657, 2003.

[126] A. W. Lowe, M. Olsen, Y. Hao et al., "Gene expression patterns in pancreatic tumors, cells and tissues," PLoS One, vol. 2, no. 3, article e323, 2007.

[127] G. L. Mutter, J. P.A. Baak, J. T. Fitzgerald et al., "Global expression changes of constitutive and hormonally regulated genes during endometrial neoplastic transformation," Gynecologic Oncology, vol. 83, no. 2, pp. 177-185, 2001.

[128] D. S. P. Tan, M. B. K. Lambros, S. Rayter et al., "PPM1D is a potential therapeutic target in ovarian clear cell carcinomas," Clinical Cancer Research, vol. 15, no. 7, pp. 2269-2280, 2009.

[129] S. Takahashi, A. Ebihara, H. Kajiho, K. Kontani, H. Nishina, and T. Katada, "RASSF7 negatively regulates pro-apoptotic JNK signaling by inhibiting the activity of phosphorylatedMKK7," Cell Death and Differentiation, vol. 18, no. 4, pp. 645-655, 2011. 

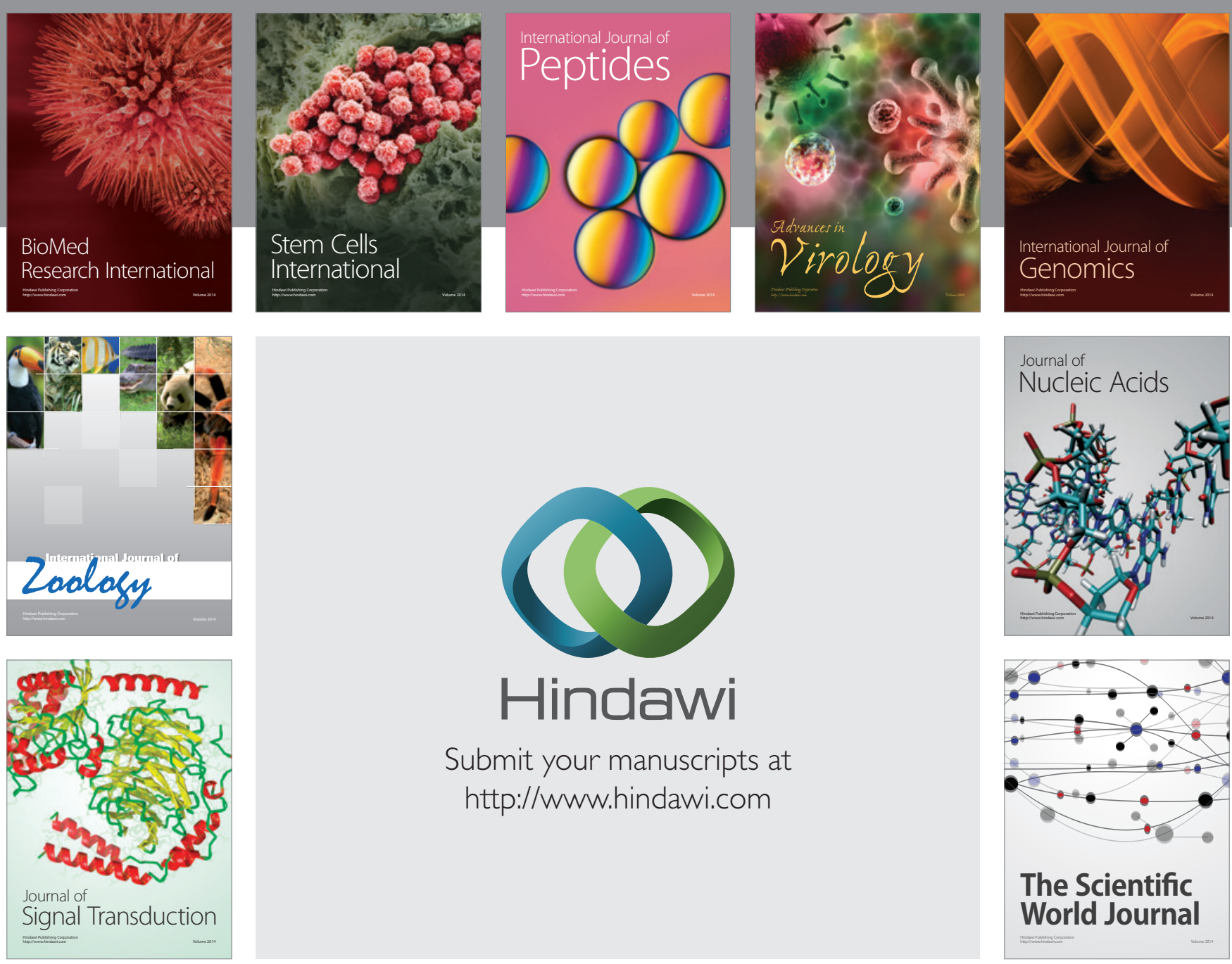

Submit your manuscripts at

http://www.hindawi.com


The Scientific World Journal
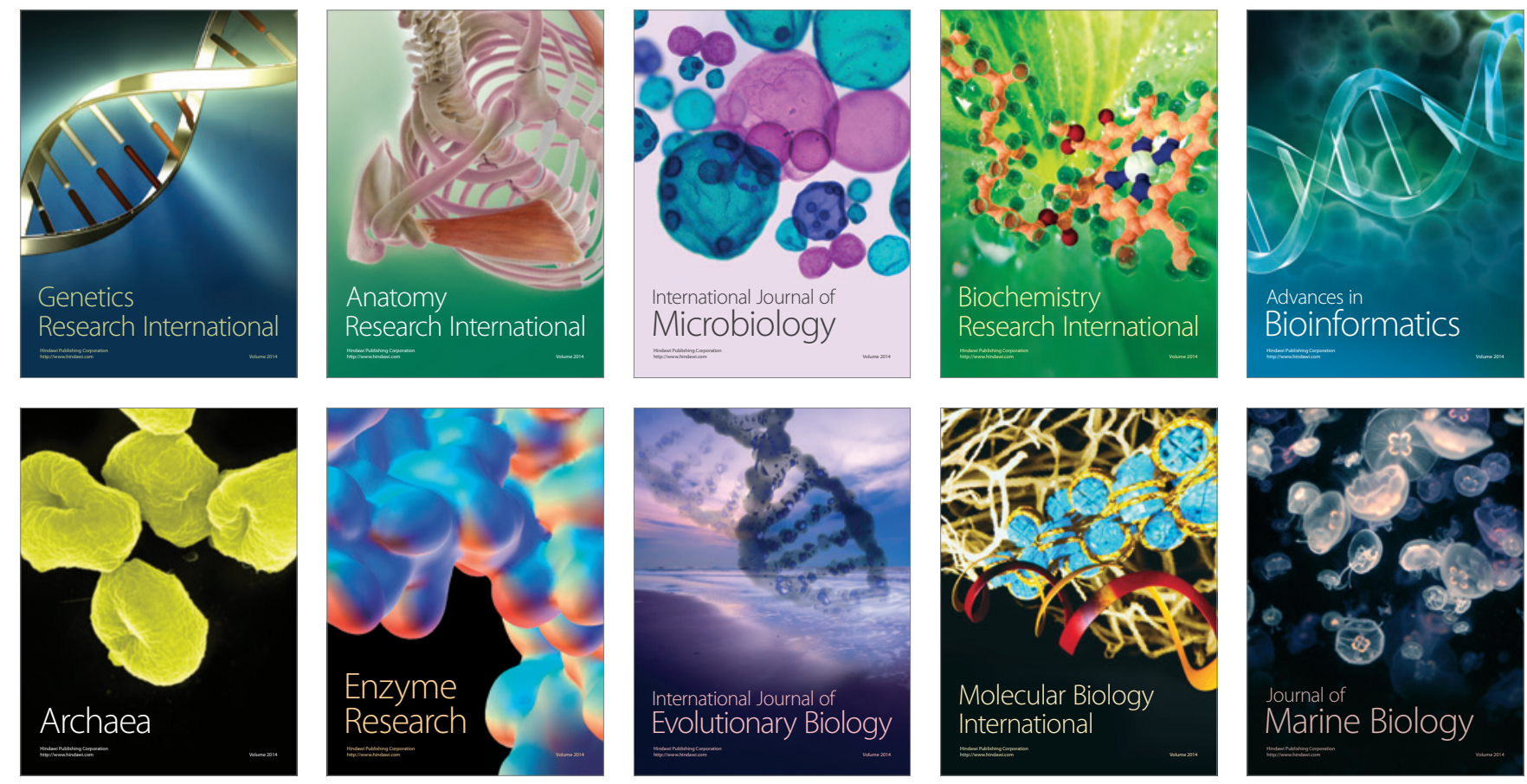